MPI-PhT/96-121

BUTP/96-27

\title{
Questionable and unquestionable in the perturbation theory of non-Abelian models
}

\author{
Ferenc Niedermayer \\ Institute for Theoretical Physics \\ University of Bern \\ Sidlerstrasse 5, CH-3012 Bern, Switzerland \\ Max Niedermaier and Peter Weisz \\ Max-Planck-Institut für Physik \\ Föhringer Ring 6, D-80805 München, Germany \\ April 2018
}

\begin{abstract}
We show, by explicit computation, that bare lattice perturbation theory in the two-dimensional $\mathrm{O}(n)$ nonlinear $\sigma$ models with superinstanton boundary conditions is divergent in the limit of an infinite number of points $|\Lambda|$. This is the analogue of David's statement that renormalized perturbation theory of these models is infrared divergent in the limit where the physical size of the box tends to infinity. We also give arguments which support the validity of the bare perturbative expansion of short-distance quantities obtained by taking the limit $|\Lambda| \rightarrow \infty$
\end{abstract}

\footnotetext{
*Work supported in part by Schweizerischer Nationalfonds

${ }^{\dagger}$ On leave from the Institute of Theoretical Physics, Eötvös University, Budapest, Hungary
} 
term by term in the theory with more conventional boundary conditions such as Dirichlet, periodic, and free. 


\section{Introduction}

Quantum chromodynamics (QCD) is the presently favored candidate theory for strong interactions. However, to establish its esteemed status the theory must be able to reproduce the low-lying spectrum and low-energy $S$-matrix elements to a respectable precision. To accomplish this we require a nonperturbative definition of the theory - the most promising of which is lattice regularization. On the other hand, many aspects of high energy phenomena involving hadrons (e.g., jets, deep inelastic scattering, etc.) are described successfully using renormalized perturbation theory (PT); the rationale being the expected property of asymptotic freedom, i.e., that the amplitudes can be expressed as a series in a coupling which depends on the energy of the process and goes to zero as the energy goes to infinity. The conventional wisdom is that "in principle" one could justify this use of PT by showing that the coefficients of the conventional perturbative series provide the true asymptotic expansion of the full theory defined nonperturbatively via the lattice regularization. Confirming this proclaimed status of PT is also essential in aiming at a criterion where to truncate it. Without such a criterion $\mathrm{PT}$ produces sequences of predictions, one for each order where one decides to truncate it, and a priori none of them might be close to the true answer. It must be emphasized, however, that the usual arguments for the above scenario are far off a proof and Patrascioiu and Seiler repeatedly emphasized that also alternative scenarios can be imagined [1, 2].

In full QCD a rigorous understanding of the status of PT is likely not to become amenable in the near future, so that it is sensible to address the question in a simpler context, e.g., the $\mathrm{O}(n)$ nonlinear $\sigma$ models in two dimensions. These models are also perturbatively asymptotically free and are thought to describe a multiplet of stable massive particles, the mass scale being nonperturbative in the coupling constant. In the lattice formulation one usually starts with a finite number of lattice points $|\Lambda|$ and the standard discretization (2.1). The conventional picture is that the critical point at which the continuum limit for $n>2$ should be taken is $\beta_{c}=\infty$. Patrascioiu and Seiler [1], on the other hand, conjecture that there is a critical $\beta_{c}<\infty$ (for all $n>1$ ) beyond which the theory is in a massless phase. It might then happen that although the continuum limit obtained by approaching $\beta_{c}$ from below has a mass gap and describes the expected low-energy properties, the theory thus obtained is not asymptotically free at high energies. In par- 
ticular, conventional perturbation theory in the infinite volume limit would then be wrong for 2-d models with a non-Abelian global symmetry. Their arguments are based on results from percolation theory [2] and with analogy to the case of the Abelian model $n=2$ (for which it is rigorously proven that there is a phase transition at finite $\beta$ [3]). Numerical investigations can give useful hints but obviously cannot establish which scenario is correct.

Concerning the status of perturbation theory it is known that for a fixed number of lattice points $|\Lambda| \sim L^{2}$ and for a large class of boundary conditions (BC's) observables have a well-defined perturbative expansion in the bare coupling $\beta^{-1}$. We shall use the term "short-distance observable" to denote $\mathrm{O}(n)$-invariant correlation functions of some local field, where (in the bulk of the paper) all arguments are fixed lattice sites, a distance $O(L)$ away from the boundary. The problems now arise with the limit $L \rightarrow \infty$. Let us first consider the following question:

Q1: Is the asymptotic expansion of short-distance observables for fixed $L$ and $\beta \rightarrow \infty$ uniform in $L$ ? Equivalently, can for such quantities the limits $\beta \rightarrow \infty$ and $L \rightarrow \infty$ be exchanged in bare lattice PT?

A major finding of [4] is that the answer to Q1 actually depends on the choice of boundary conditions. The reasoning can be illustrated with the computation of the energy density $\mathcal{E}$ at the center of the lattice. Although $\mathcal{E}$ does not have a continuum limit relevant to the physics, by application of the Mermin-Wagner theorem [5] it should be independent of the boundary conditions in the limit of an infinite number of lattice points. Patrascioiu and Seiler invented boundary conditions (which they coined superinstanton (SI) boundary conditions; c.f. sect. 2) and computed the one-loop coefficient of $\mathcal{E}$. They showed that it has a finite limit as $L \rightarrow \infty$ but the result was different from that with more conventional BC's such as Dirichlet. Furthermore they claim a similar result holds for the renormalization group $\beta$-functions. The conclusion is that for at least one of the BC's involved the answer to Q1 is negative. The authors interpret their result as casting doubt on the validity of standard PT for all BC's. They claim that the correct perturbative treatment in the limit of infinite volume must include expansions around the so-called superinstanton gas (configurations whose energy tends to zero as $L \rightarrow \infty$; see sect. 3 ). 
We believe that such conclusions are too strong and we set out to clarify some of the points raised. In the next section we show that the SI BC are "sick" in the sense that the limits in Q1 cannot be interchanged. We do this by showing that the perturbative coefficients of $\mathcal{E}$ with SI BC diverge at two-loop order in the limit $L \rightarrow \infty$.] We have not considered the particular $\beta$-functions defined in [1] but we believe a similar result will be found. This infrared divergence of the PT with SI BC was already suspected by David [6] but as his argument was formulated in the framework of (renormalized) continuum PT his claims were open to a counterattack [7] and the issue remained unsettled. Our result can be viewed as the lattice analogue of David's statement.

Having identified the SI BC as "sick", the next question is whether the conventional BC are "healthy". Consider the bare lattice PT expansion of some short-distance observable on a finite lattice $|\Lambda| \sim L^{2}$ with various BC's. Let $c_{r}^{\alpha}\left(x_{1}, \ldots, x_{n} ; L\right), r \geq 1$ be the coefficient of $\beta^{-r}$ in an asymptotic expansion with $\mathrm{BC}$ of type $\alpha$, where the sites $x_{1}, \ldots, x_{n}$ are fixed and a distance $\mathrm{O}(L)$ away from the boundary.

Q2: Which BC's $\alpha$ can be anticipated to give finite and coinciding answers for the $L \rightarrow \infty$ limits of their perturbative coefficients $c_{r}^{\alpha}\left(x_{1}, \ldots, x_{n} ; L\right)$ ?

Q3: Do the infinite volume short-distance observables admit an asymptotic expansion in $\beta^{-1}$ ? If "yes", can their coefficients $c_{r}^{\infty}\left(x_{1}, \ldots, x_{n}\right), r \geq 1$ be obtained from PT via $\lim _{L \rightarrow \infty} c_{r}^{\alpha}\left(x_{1}, \ldots, x_{n} ; L\right)=c_{r}^{\infty}\left(x_{1}, \ldots, x_{n}\right)$, where $\alpha$ is one of the $\mathrm{BC}$ meeting the conditions in Q2?

In section 3 we shall consider these deeper questions raised in [1, 4] and argue in favor of the following picture: (i) All BC's involving only ferromagnetic couplings and leaving the interior spins unconstrained meet the condition in Q2 - provided free and Dirichlet BC meet it. (ii) Assuming the latter also the answer to both parts of $\mathbf{Q 3}$ is affirmative. We do not have a rigorous theorem but our considerations may constitute a strategy for a future proof thereof. Section 4 is devoted to some further discussion.

\footnotetext{
${ }^{1}$ We have verified, however, that if we compute quantities well away from the boundaries and the center then the same results are obtained as for conventional BC.
} 


\section{IR divergence of PT with superinstanton BC}

We consider a two-dimensional square lattice $\Lambda=\left\{\left(x_{1}, x_{2}\right) ;-L / 2+1 \leq\right.$ $\left.x_{k} \leq L / 2-1\right\}, L$ a positive even integer. The set of points surrounding the square, $\left\{\left|x_{1}\right|=L / 2,\left|x_{2}\right| \leq L / 2\right\} \cup\left\{\left|x_{1}\right| \leq L / 2,\left|x_{2}\right|=L / 2\right\}$ will be referred to as the boundary $\partial \Lambda$ of $\Lambda$. The $\mathrm{O}(n)$ spin field $\mathbf{S}_{x}$ defined on $\Lambda \cup \partial \Lambda$ is an $n$-component field of unit length $\mathbf{S}_{x}^{2}=1$. We restrict attention to the standard lattice action

$$
\mathcal{A}(S)=\beta \sum_{x, \mu}\left(1-\mathbf{S}_{x} \cdot \mathbf{S}_{x+\mu}\right) .
$$

For fixed $L$ the perturbative expansion of the two-point function is

$$
C^{\alpha}(x, y ; \beta, L) \equiv\left\langle\mathbf{S}_{x} \cdot \mathbf{S}_{y}\right\rangle=1-\sum_{r \geq 1} \beta^{-r} c_{r}^{\alpha}(x, y ; L),
$$

where the superscript $\alpha$ indicates the dependence on the boundary conditions under consideration. In this section we will only consider two sorts of BC's, the Dirichlet boundary condition $S_{x}^{a}=\delta_{a n}$ for $x \in \partial \Lambda$ and the socalled superinstanton (SI) boundary conditions 淊. The latter are Dirichlet BC's with the additional constraint that the field at some point $z_{0}$ is also held fixed parallel to the fields at the boundary.

The purpose of this section is to show, by explicit computation, that PT with SI BC's is infrared (IR) divergent at third order, while PT with Dirichlet or periodic BC is IR finite at the same order. For completeness, and to introduce the notations, we first reconsider the first and second orders in some detail. Bare perturbation theory can be performed by parametrizing the fields by $S_{x}^{a}=\beta^{-\frac{1}{2}} \pi_{x}^{a}, a=1, \ldots, N$ where $N=n-1$, and $S_{x}^{n}=\left(1-\beta^{-1} \vec{\pi}_{x}^{2}\right)^{\frac{1}{2}}$. The measure $\Pi_{x \in \Lambda} d \mathbf{S}_{x} \delta\left(\mathbf{S}_{x}^{2}-1\right)$ is then replaced by $\exp \left(-\mathcal{A}_{\text {measure }}\right) \Pi_{x \in \Lambda} d \vec{\pi}_{x}$ with $\mathcal{A}_{\text {measure }}=\frac{1}{2} \sum_{x} \ln \left(1-\beta^{-1} \vec{\pi}_{x}^{2}\right)$. The Feynman diagrams contributing to the two-point function are the same for the two BC's, only the expression for the free propagator differs. We denote the free Dirichlet propagator by $D(x, y)$ and the free SI propagator by $G(x, y)$. One has

$$
G(x, y)=D(x, y)-\frac{D\left(x, z_{0}\right) D\left(z_{0}, y\right)}{D\left(z_{0}, z_{0}\right)} .
$$

\footnotetext{
${ }^{2}$ This is not indispensable, a parametrization independent definition could be given via the Schwinger-Dyson equations.
} 
In the following we will take the point $z_{0}$ to be the origin $z_{0}=(0,0)$. Also we denote by $z_{1}$ the point $(1,0)$. One easily sees that each $c_{r}^{\alpha}(x, y ; L)$ is a polynomial in $N=n-1$ of degree $r$ or less, and we write

$$
c_{r}^{\alpha}=\sum_{j=1}^{r} c_{r ; j}^{\alpha} N^{j} .
$$

\section{$2.1 \quad$ First order}

In first order we have, for the Dirichlet BC,

$$
c_{1}^{\text {Dir }}(x, y)=\frac{1}{2} N[D(x, x)+D(y, y)-2 D(x, y)] .
$$

As mentioned above, for the SI BC one simply replaces $D$ by $G$. For the special case of the energy expectation value we have

$$
c_{1}^{\operatorname{Dir}}\left(z_{0}, z_{1}\right)=N\left(\frac{1}{4}-\frac{1}{2} \triangle_{0}\right)
$$

where

$$
\triangle_{0}=D\left(z_{0}, z_{0}\right)-D\left(z_{1}, z_{1}\right) .
$$

$c_{1}^{\text {Dir }}$ approaches its asymptotic value with power corrections since $\triangle_{0}=$ $O\left(1 / L^{2}\right)$ for $L \rightarrow \infty$. On the other hand

$$
c_{1}^{\mathrm{SI}}\left(z_{0}, z_{1}\right)=N \frac{1}{2} G\left(z_{1}, z_{1}\right)
$$

approaches the same asymptotic value extremely slowly with corrections $1 / \ln L$ since

$$
G\left(z_{1}, z_{1}\right)=\frac{1}{2}-\triangle_{0}-\frac{1}{16} Z^{-1}
$$

with

$$
Z \equiv D\left(z_{0}, z_{0}\right) \sim(2 \pi)^{-1} \ln L, \quad \text { for } L \rightarrow \infty
$$

\subsection{Second order}

At the next order there are three contributions

$$
c_{2}^{\alpha}(x, y)=\sum_{r=1}^{3} c_{2}^{\alpha(r)}(x, y)
$$




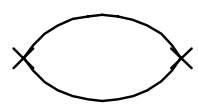

1

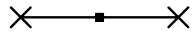

2

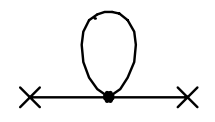

3

Figure 1: One-loop diagrams contributing to the spin-spin correlation function

corresponding to the diagrams depicted in Fig. 1 (diagram 2 comes from the measure part of the action). One has

$$
\begin{aligned}
& c_{2}^{\text {Dir }}{ }^{(1)}(x, y)=\frac{1}{8} N^{2}[D(x, x)-D(y, y)]^{2} \\
& +\frac{1}{4} N\left[D(x, x)^{2}+D(y, y)^{2}-2 D(x, y)^{2}\right] \text {, } \\
& c_{2}^{\operatorname{Dir}{ }^{(2)}}(x, y)=\frac{1}{2} N \sum_{i} \widetilde{D}(x, y ; i)^{2},
\end{aligned}
$$

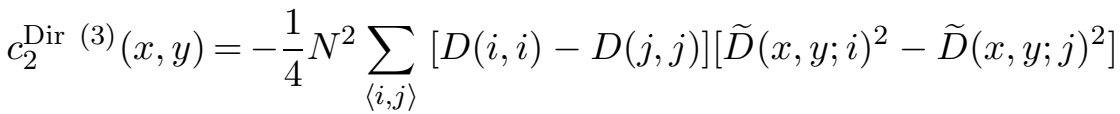

$$
\begin{aligned}
& -\frac{1}{2} N \sum_{\langle i, j\rangle}\{\widetilde{D}(x, y ; i)[D(i, i) \widetilde{D}(x, y ; i)-D(i, j) \widetilde{D}(x, y ; j)] \\
& +(i \leftrightarrow j)\}
\end{aligned}
$$

where the sums in the last equation are restricted to the case when $i$ and $j$ are nearest neighbors and we have introduced the notation

$$
\widetilde{D}(x, y ; i)=D(x, i)-D(y, i) .
$$

Let us just inspect the coefficient of $N^{2}$. Here diagram 2 does not contribute and for diagram 1 one has simply

$$
c_{2 ; 2}^{\mathrm{SI}}{ }^{(1)}\left(z_{0}, z_{1}\right)-c_{2 ; 2}^{\mathrm{Dir}}{ }^{(1)}\left(z_{0}, z_{1}\right)=\frac{1}{8}\left[G\left(z_{1}, z_{1}\right)^{2}-\triangle_{0}^{2}\right]
$$

which by eq. (2.8) tends to $1 / 32$ as $L \rightarrow \infty$. For diagram 3 we find

$$
c_{2 ; 2}^{\mathrm{SI}}{ }^{(3)}\left(z_{0}, z_{1}\right)-c_{2 ; 2}^{\operatorname{Dir}}{ }^{(3)}\left(z_{0}, z_{1}\right)=\frac{1}{16} \triangle_{0}+t_{1} Z^{-1}+t_{2} Z^{-2}+t_{3} Z^{-3},
$$


with

$$
\begin{aligned}
t_{1} & =\frac{1}{4} \sum_{i \neq z_{0}} \widetilde{D}\left(z_{0}, z_{1} ; i\right)^{2} E(i), \\
t_{2} & =-\frac{1}{64} \sum_{i \neq z_{0}} D\left(z_{0}, i\right)^{2} F(i), \\
t_{3} & =\frac{1}{64} \sum_{i \neq z_{0}} D\left(z_{0}, i\right)^{2} E(i),
\end{aligned}
$$

where

$$
\begin{aligned}
& E(i)=\sum_{j=\langle i\rangle}\left[D\left(i, z_{0}\right)^{2}-D\left(j, z_{0}\right)^{2}\right], \\
& F(i)=\sum_{j=\langle i\rangle}[D(i, i)-D(j, j)] .
\end{aligned}
$$

Here the sums are taken over sites $j$ which are nearest neighbors to $i$. The decomposition in the form of eq. (2.13) is made in order to be able to extract the asymptotic behavior reliably. Our ansatz (which we have not proven analytically) is that the functions $t_{i}$ have an expansion of the form

$$
t_{i}(L) \sim \sum_{r=0}^{R_{i}} t_{i}^{(r)}\left[(2 \pi)^{-1} \ln L\right]^{r}+O(1 / L),
$$

with $R_{i}$ finite. To obtain the leading behavior we have computed $t_{i}(L)$ over a large range of $L$ (up to $L=220$, using extended precision arithmetics) and taken logarithmic derivatives with respect to $L$. Our findings are that

$$
R_{1}=R_{2}=0,
$$

and hence the contributions in eq. (2.13) involving $t_{1}, t_{2}$ vanish in the limit $L \rightarrow \infty$. However,

$$
R_{3}=3, \quad t_{3}^{(3)}=-\frac{1}{96} .
$$

One can in fact understand the result for $t_{3}$ analytically in the following way. Far from the origin and the boundary, i.e., for $1 \ll|i| \ll L$ the Dirichlet propagator is well approximated by its continuum behavior

$$
D\left(z_{0}, i\right) \sim(2 \pi)^{-1} \ln \frac{L}{|i|},
$$


and so

$$
E(i) \sim-\square_{(i)}\left(D\left(z_{0}, i\right)^{2}\right) \sim-2(2 \pi)^{-2}|i|^{-2},
$$

where $\square_{(i)}$ is the laplacian in the variable $i$. Thus for $t_{3}$ we have

$$
\begin{aligned}
t_{3} & \sim-\frac{1}{32}(2 \pi)^{-4} \frac{1}{L^{2}} \sum_{i \neq z_{0}}\left(\frac{L}{|i|}\right)^{2} \ln ^{2} \frac{L}{|i|} \\
& \sim-\frac{1}{32}(2 \pi)^{-4} \int_{|x|>1 / L}^{|x|=1} \mathrm{~d}^{2} x|x|^{-2} \ln ^{2}|x|=-\frac{1}{96}(2 \pi)^{-3} \ln ^{3} L .
\end{aligned}
$$

This is the same result as was guessed in [4]. These authors were quite fortunate to get the correct result because initially they just evaluated the full function $c_{2 ; 2}^{\mathrm{SI}}{ }^{(3)}$ numerically and then made a rather naive extrapolation. We stress that the analysis has to be done extremely carefully on the lines outlined above to properly treat the appearance of polynomials of logarithms in the denominators.

The final result of this subsection is that

$$
\lim _{L \rightarrow \infty}\left[c_{2 ; 2}^{\mathrm{SI}}\left(z_{0}, z_{1}\right)-c_{2 ; 2}^{\mathrm{Dir}}\left(z_{0}, z_{1}\right)\right]=\frac{1}{48}
$$

(we also know $\lim _{L \rightarrow \infty} c_{2 ; 2}^{\operatorname{Dir}}\left(z_{0}, z_{1}\right)=0$ ). The Mermin-Wagner theorem implies that the perturbative expansion of the two-point function is independent of the boundary conditions in the infinite volume limit. Accepting this fact we must conclude that the interchange of the limits $\beta \rightarrow \infty$ and $L \rightarrow \infty$ is not permissible for at least one of the boundary conditions involved. We now go on to show that this is indeed the case for the SI BC because one encounters an infrared divergence.

\subsection{Leading $N$ contribution to the third order}

For the purpose of showing an IR divergence at some order $r$, for generic $n$, it is sufficient to show that one of the coefficients of this polynomial in $N$ is IR divergent. We claim that this is the case for the coefficient of $N^{3}$ in $c_{3}^{\alpha}(x, y ; L)$ with the SI BC.

${ }^{3}$ Indeed, $c_{2 ; 2}^{\mathrm{SI}}{ }^{(3)}$ is not monotonic in $L$ and has a minimum at a large value of $L \sim 120$. 

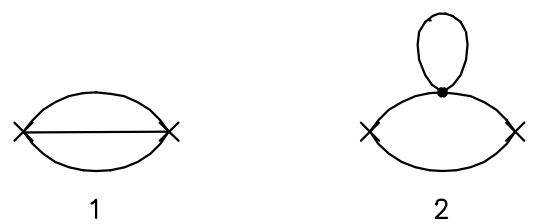

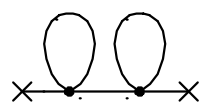

3

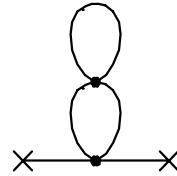

4

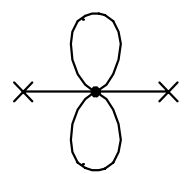

Figure 2: Two-loop diagrams contributing to the spin-spin correlation function with terms $\propto N^{3}$

Out of 12 diagrams only the 5 shown in Fig. 2 have a nonvanishing $O\left(N^{3}\right)$ coefficient. Explicitly

$$
c_{3 ; 3}^{\mathrm{SI}}(x, y ; L)=\sum_{r=1}^{5} c_{3 ; 3}^{\mathrm{SI}}{ }^{(r)}(x, y ; L),
$$

with

$$
\begin{aligned}
& c_{3 ; 3}^{\mathrm{SI}}{ }^{(1)}=\frac{1}{16}[G(x, x)-G(y, y)]^{2}[G(x, x)+G(y, y)], \\
& c_{3 ; 3}^{\mathrm{SI}}{ }^{(2)}=-\frac{1}{8}[G(x, x)-G(y, y)] \sum_{\langle i, j\rangle}[G(i, i)-G(j, j)] \\
& \times\left[G(x, i)^{2}-G(y, i)^{2}-G(x, j)^{2}+G(y, j)^{2}\right], \\
& c_{3 ; 3}^{\mathrm{SI}}{ }^{(3)}=\frac{1}{8} \sum_{\langle i, j\rangle\langle k, l\rangle}\{[G(i, i)-G(j, j)][G(k, k)-G(l, l)] \\
& \times G(i, k) \widetilde{G}(x, y ; i) \widetilde{G}(x, y ; k)+3 \text { perms }\}, \\
& c_{3 ; 3}^{\mathrm{SI}}{ }^{(4)}=\frac{1}{8} \sum_{\langle i, j\rangle\langle k, l\rangle}\left\{\left[\widetilde{G}(x, y ; i)^{2}-\widetilde{G}(x, y ; j)^{2}\right]\right.
\end{aligned}
$$




$$
\begin{gathered}
\left.\times[G(k, k)-G(l, l)] G(i, k)^{2}+3 \text { perms }\right\} \\
c_{3 ; 3}^{\text {SI }}(5)=-\frac{1}{16} \sum_{\langle i, j\rangle}\left\{\widetilde{G}(x, y ; i)^{2}[G(i, i)-G(j, j)][3 G(i, i)+G(j, j)]\right. \\
+(i \leftrightarrow j)\} .
\end{gathered}
$$

Here " +3 perms" stands for three similar terms with summation indices permuted according to " $+(i \leftrightarrow j)+(k \leftrightarrow l)+(i \leftrightarrow j, k \leftrightarrow l)$ ".

Again we restrict attention to the energy expectation value at the center of the lattice. First we note that for the analogous Dirichlet expression one can show $\lim _{L \rightarrow \infty} c_{3 ; 3}^{\text {Dir }}\left(z_{0}, z_{1}\right)=0$. For the SI functions we again insert the formula, eq. (2.3), for $G$ and separate the coefficients of $Z^{-r}$.

Using the results in the last subsection together with the knowledge that $\lim _{L \rightarrow \infty} \sum_{i \neq z_{0}} \widetilde{D}\left(z_{0}, z_{1} ; i\right)^{2} F(i)=0$ it is easy to show that the contributions from diagrams 1 and 2 in Fig. 2 have finite limits as $L \rightarrow \infty$. It turns out that all the remaining diagrams $3-5$ in Fig. 2 diverge logarithmically. Consider first diagrams 3 and 4. Dropping all terms which have finite $L \rightarrow \infty$ limits one obtains the decomposition

$$
\begin{aligned}
c_{3 ; 3}^{\mathrm{SI}}{ }^{(3)}\left(z_{0}, z_{1}\right) & \sim s_{4} Z^{-4}-32 t_{3}^{2} Z^{-5} \\
c_{3 ; 3}^{\mathrm{SI}}{ }^{(4)}\left(z_{0}, z_{1}\right) & \sim s_{2} Z^{-2}+s_{3} Z^{-3}+2 s_{4} Z^{-4}-32 t_{3}\left(t_{3}+t_{4} Z^{2}\right) Z^{-5}(2 .
\end{aligned}
$$

where $t_{4}$ is a one-loop sum

$$
t_{4}=\frac{1}{4} \sum_{i \neq z_{0}} D\left(z_{0}, i\right)^{2} H(i)
$$

with

$$
H(i)=\sum_{j=\langle i\rangle}\left[\widetilde{D}\left(z_{0}, z_{1} ; i\right)^{2}-\widetilde{D}\left(z_{0}, z_{1} ; j\right)^{2}\right] .
$$

The $s_{r}$ are more complicated two-loop sums

$$
\begin{aligned}
s_{2} & =\frac{1}{4} \sum_{i \neq z_{0}} \sum_{k \neq z_{0}} H(i) E(k) D(i, k) D\left(z_{0}, i\right) D\left(z_{0}, k\right), \\
s_{3} & =-\frac{1}{128} \sum_{i \neq z_{0}} \sum_{k \neq z_{0}} E(i) E(k) D(i, k)^{2}, \\
s_{4} & =\frac{1}{128} \sum_{i \neq z_{0}} \sum_{k \neq z_{0}} E(i) E(k) D(i, k) D\left(z_{0}, i\right) D\left(z_{0}, k\right) .
\end{aligned}
$$


We have numerically computed all these sums over a large range of $L$ (up to $L=210$ ) and their asymptotic behavior as $L \rightarrow \infty$ was determined using extrapolation techniques described in the previous subsection. In all cases we found results consistent with those obtained by substituting the behaviors of the functions at large separations:

$$
\begin{aligned}
t_{4} & \sim \frac{1}{32} Z, & s_{2} & \sim-\frac{1}{96} Z^{3}, \\
s_{3} & \sim-\frac{1}{192} Z^{4}, & s_{4} & \sim \frac{1}{240} Z^{5} .
\end{aligned}
$$

We do not give all the derivations but illustrate the procedure again with the interesting case of $s_{4}$. Apart from the approximations (2.19), (2.20) we introduce $D(i, k) \sim(1 / 2 \pi) \ln (L /|i-k|)$ so that

$$
s_{4} \sim \frac{1}{32}(2 \pi)^{-7} \sum_{i \neq z_{0}} \sum_{k \neq z_{0}} \frac{1}{i^{2} k^{2}} \ln \frac{L}{|i|} \ln \frac{L}{|k|} \ln \frac{L}{|i-k|} .
$$

Now by symmetry we can consider $|k|<|i|$ and averaging first over the angle between $i$ and $k$ we obtain

$$
\begin{aligned}
s_{4} & \sim \frac{1}{16}(2 \pi)^{-7} \sum_{i \neq z_{0}} \frac{1}{i^{2}} \ln \frac{L}{|i|} \int_{|i| \geq|k| \geq 1} \mathrm{~d}^{2} k \frac{1}{k^{2}} \ln \frac{L}{|k|} \ln \frac{L}{|i-k|} \\
& \sim \frac{1}{16}(2 \pi)^{-6} \sum_{i \neq z_{0}} \frac{1}{i^{2}} \ln ^{2} \frac{L}{|i|} \int_{1}^{|i|} \frac{d r}{r} \ln \frac{L}{r} \\
& =\frac{1}{32}(2 \pi)^{-6} \sum_{i \neq z_{0}} \frac{1}{i^{2}} \ln ^{2} \frac{L}{|i|}\left[\ln ^{2} L-\ln ^{2} \frac{L}{|i|}\right] \\
& \sim \frac{1}{32}(2 \pi)^{-5}\left[\frac{1}{3}-\frac{1}{5}\right] \ln ^{5} L=\frac{1}{240}(2 \pi)^{-5} \ln ^{5} L .
\end{aligned}
$$

The corresponding numerical result is shown in Fig. 3. The fifth logarithmic derivative of $s_{4}$ multiplied by $(2 \pi)^{5} / 5$ ! is shown as a function of $1 / L$. The definition of the lattice logarithmic derivative used is

$$
L \frac{\partial}{\partial L} f(L)=\frac{L}{2}[f(L+1)-f(L-1)]
$$

The data are calculated for even values of $L$ in the ranges $8-60,100-120$, 200-210 using extended precision arithmetic. The dotted line is the analytic result $1 / 240$. 


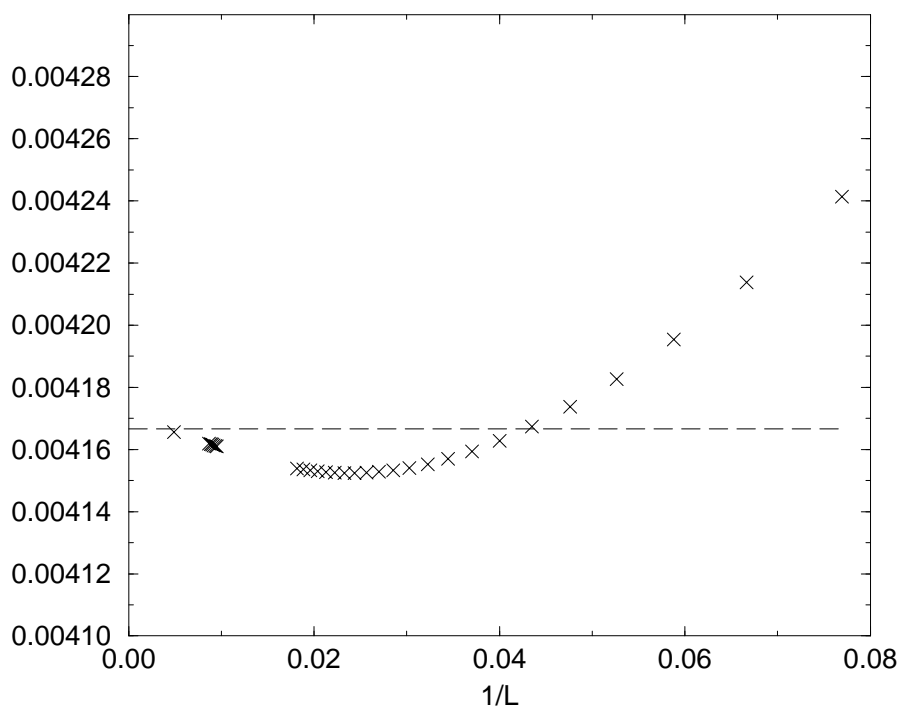

Figure 3: The 5 -th logarithmic derivative of $s_{4}$ multiplied by $(2 \pi)^{5} / 5$ ! plotted versus $1 / L$.

The coefficient $c_{3 ; 3}^{\mathrm{SI}}{ }^{(5)}\left(z_{0}, z_{1}\right)$ involves new sums but can be treated similarly. Summarizing we find

$$
\begin{aligned}
c_{3 ; 3}^{\mathrm{SI}}{ }^{(3)}\left(z_{0}, z_{1}\right) & \sim \frac{1}{1440} Z, \\
c_{3 ; 3}^{\mathrm{SI}}{ }^{(4)}\left(z_{0}, z_{1}\right) & \sim-\frac{1}{2880} Z, \\
c_{3 ; 3}^{\mathrm{SI}}{ }^{(5)}\left(z_{0}, z_{1}\right) & \sim \frac{1}{480} Z .
\end{aligned}
$$

Thus the divergent contributions do not cancel and one ends up with an overall logarithmic divergence $c_{3 ; 3}^{\mathrm{SI}}\left(z_{0}, z_{1}\right) \sim(7 / 2880) Z$ as $L \rightarrow \infty$.

This shows that one cannot interchange the limits $\beta \rightarrow \infty$ and $L \rightarrow \infty$ for the SI BC because the perturbative coefficients diverge at two-loop order. In particular, the SI BC's do not belong to the class of "healthy" ones alluded to in Q2 and Q3 cannot even be addressed. Initially however, 
the SI example was probably thought to discredit PT on internal grounds, stemming from the apparent ambiguities in the PT answers for the infinite lattice quantities 泊. As such, we think, it is no longer valid. Of course one could still argue that the divergent SI answer might even hit a point in signaling that the quantities in question do not have an asymptotic expansion in $\beta^{-1}$. However this possibility is not an argument by itself and has to be supported by external means, see, e.g., [2]. In the next section we shall leave this example and try to develop criteria of what healthy BC's should look like and under what conditions an affirmative answer to Q3 should be expected.

\section{Addressing the questions Q2 and Q3}

We start by comparing the correlator between two spins for different boundary conditions. The discussion will be based on the validity of the following conjecture: For a spin system with ferromagnetic couplings the correlation function $C(x, y)=\left\langle\mathbf{S}_{x} \cdot \mathbf{S}_{y}\right\rangle$ increases if any of the ferromagnetic couplings is increased. This conjecture is physically rather intuitive, nevertheless it has not been proven yet [8]. Consider a spin system with a given ferromagnetic interaction on:

1. a finite lattice $\Lambda$ with free boundary conditions,

2. the same lattice with Dirichlet BC, and

3. an infinite lattice.

Validity of the above conjecture implies the following inequalities between the corresponding correlators:

$$
C^{\text {free }}(x, y ; \beta, \Lambda) \leq C^{\infty}(x, y ; \beta) \leq C^{\text {Dir }}(x, y ; \beta, \Lambda),
$$

for any $x, y \in \Lambda$. More generally, the conjecture also implies that on any lattice $\Lambda^{*}$ containing $\Lambda \cup \partial \Lambda$ the correlator $C^{*}\left(x, y ; \beta, \Lambda^{*}\right)$ satisfies

$$
C^{\text {free }}(x, y ; \beta, \Lambda) \leq C^{*}\left(x, y ; \beta, \Lambda^{*}\right) \leq C^{\operatorname{Dir}}(x, y ; \beta, \Lambda) .
$$


From here it follows that $C^{\text {free }}\left(x, y ; \beta, \Lambda^{*}\right)$ increases while $C^{\operatorname{Dir}}\left(x, y ; \beta, \Lambda^{*}\right)$ decreases with increasing size $\left|\Lambda^{*}\right|$. Further the systems with periodic BC on $\Lambda^{*}$ will satisfy eq. (3.2). Note that the SI BC with $z_{0} \in \Lambda$ (where $z_{0}$ is the site where the extra spin is fixed) does not belong to the class considered in eq. (3.2), the considerations apply, however, when $z_{0}$ is outside $\Lambda$.

In the following we shall argue that if the correlation functions of the systems 1 and 2 have well-behaved perturbative expansions - in a sense to be specified below - then

(i) the infinite system 3 has an asymptotic expansion with coefficients given by the formal $L \rightarrow \infty$ limit of the perturbative coefficients of systems 1 and 2, and

(ii) all systems considered under eq. (3.2) have similarly well-behaved perturbative expansions.

For this purpose we have to make some natural and verifiable assumptions on the perturbative series with free and Dirichlet BC's. To simplify the notation we shall return to the square lattice of section 2 and write $C^{\alpha}(\beta, L)$ for $C^{\alpha}(x, y ; \beta, \Lambda)$. The distance of the fixed sites $x, y$ from the boundary is taken to be $\mathrm{O}(L)$, so that $C^{\alpha}(\beta, L)$ qualifies as a short-distance observable, as defined in the Introduction.

Let us elucidate on what we mean by a "well-behaved" perturbative expansion. For fixed $L$ and given boundary conditions $\alpha=$ free or Dirichlet, one can calculate $C^{\alpha}$ in $\mathrm{PT}$ as a power series in $1 / \beta$ to some arbitrary order $k$ :

$$
C^{\alpha}(\beta, L)=1-\sum_{r=1}^{k} c_{r}^{\alpha}(L) \beta^{-r}+R_{k}^{\alpha}(\beta, L) .
$$

There is perhaps no doubt that for fixed $L$ these are asymptotic series, i.e.

$$
R_{k}^{\alpha}(\beta, L)=\mathrm{o}\left(\beta^{-k}\right)
$$

Consider now the formal $L \rightarrow \infty$ limit of the coefficients $c_{r}^{\alpha}(L)$. On the grounds of (the lattice counterpart of) David's analysis [9] one expects that for the case of the free, Dirichlet (and periodic) BC's there are no infrared singularities and that the convergence to the $L \rightarrow \infty$ limit is of $\mathrm{O}(1 / L)$ (up 
to logarithms, i.e. $\left.\mathrm{O}\left(1 / L \cdot \ln ^{k} L\right)\right)$. We shall assume that the perturbative coefficients obtained actually coincide in the infinite volume limit:

$$
c_{r}^{\alpha}(L)=\bar{c}_{r}+\mathrm{O}\left(\frac{1}{L}\right), \quad \alpha=\text { free, Dirichlet }
$$

provided the distance of the fixed sites $x, y$ from the boundary is $\mathrm{O}(L)$. One has good reason to trust (3.5). The free propagator for these and other conventional $\mathrm{BC}$ has a size dependence of $\mathrm{O}\left(1 / L^{2}\right)$ in contrast to the SI case with an $\mathrm{O}(1 / \ln L)$ dependence. Thus conventional boundary conditions should not produce such peculiar behavior as observed in the one-loop order for the SI BC's. Assumption (3.5) can in principle be verified order by order. It could also be relaxed [8] (see note added), but we expect eq. (3.5) to describe the form of the actual finite size corrections. For $r=1,2$ we have verified this numerically. Note that $r=2$ is already the source of troubles in the SI case.

Clearly, the problem of establishing the claims (i) and (ii) amounts to controlling the remainders in an ansatz for an asymptotic expansion of $C^{\infty}(\beta)$ or for the correlators in eq. (3.2). Let us first consider claim (i) and define

$$
R_{k}^{\infty}(\beta)=C^{\infty}(\beta)-1+\sum_{r=1}^{k} \bar{c}_{r} \beta^{-k} .
$$

This definition is chosen such that claim (i) is equivalent to the statement $R_{k}^{\infty}(\beta)=o\left(\beta^{-k}\right), k \geq 1$. That is $C^{\infty}(\beta)$ has an asymptotic expansion in powers of $\beta^{-1}$ and its coefficients coincide with $\bar{c}_{r}$. For later use let us also introduce

$$
A_{k}^{\alpha}(\beta, L)=\sum_{r=1}^{k}\left[c_{r}^{\alpha}(L)-\bar{c}_{r}\right] \beta^{-r}
$$

and note that one can now rewrite (3.1) in the form

$$
A_{k}^{\text {free }}(\beta, L)+R_{k}^{\text {free }}(\beta, L) \leq R_{k}^{\infty}(\beta) \leq A_{k}^{\text {Dir }}(\beta, L)+R_{k}^{\text {Dir }}(\beta, L) .
$$

The naive strategy to establish (i) would be to first try to show that $\left|R_{k}^{\alpha}(\beta, L)\right|, \alpha=$ free, Dir are bounded by some $L$-independent function of $o\left(\beta^{-k}\right)$ and then conclude from eq. (3.8) that $R_{k}^{\infty}(\beta)=o\left(\beta^{-k}\right)$. Our main technical observation is that one can reach the same conclusion using a bound on $\left|R_{k}^{\alpha}(\beta, L)\right|$ which is much less stringent, and which needs to be 
established only in some part of the $L$ - $\beta$ plane. In detail, we assume that the bound

$$
\left|R_{k}^{\alpha}(\beta, L)\right| \leq B_{k} \frac{(\ln L)^{p(k)}}{\beta^{k+1}},
$$

with some finite $B_{k}$ and $p(k) \geq 0$ has been established in the following region of the $L$ - $\beta$ plane

$$
L \leq L_{1}(\beta), \beta>\beta_{0} \text { where }\left(\ln L_{1}(\beta)\right)^{p(k)} / \beta \rightarrow 0 \text { as } \beta \rightarrow \infty
$$

and $\beta_{0}$ is some (L-independent) constant. Convenient choices for the function $L_{1}(\beta)$ will be given below. Similarly define another region of the $L-\beta$ plane by

$$
L_{0}(\beta) \leq L, \beta>\beta_{0} \quad \text { where } \beta^{k-1} / L_{0}(\beta) \rightarrow 0 .
$$

Choosing now any unbounded path $L(\beta)$ in the intersection of both regions $(3.10)$ and $(3.11)$ the desired conclusion can be reached immediately: One has $A_{k}^{\alpha}(\beta, L(\beta))=o\left(\beta^{-k}\right)$ by eq. (3.5) and the condition on $L_{0}(\beta)$. Similarly from eq. (3.9) and the condition on $L_{1}(\beta)$ it follows that $R_{k}^{\alpha}(\beta, L(\beta))=$ $o\left(\beta^{-k}\right)$. Then the inequalities (3.8) imply

$$
R_{k}^{\infty}(\beta)=o\left(\beta^{-k}\right),
$$

which is basically the required result. Strictly speaking, one should for a fixed $k$ refer to the numbers $\bar{c}_{r}, r \leq k$ as the (unique) candidates for the coefficients in an asymptotic expansion of $C^{\infty}(\beta)$ in $\beta^{-1}$. Only after eq. (3.9) and hence eq. (3.12) has been shown for all $k$, the existence of the asymptotic expansion follows and the numbers $\bar{c}_{r}$ can properly be referred to as the coefficents of this expansion, so that claim (i) follows. It is also easy to see that starting with eq. (3.2) the same strategy applies and eq. (3.9) also implies claim (ii).

Let us add a number of comments. First one should check that choices for $L_{0}(\beta)$ and $L_{1}(\beta)$ exist such that the intersection of the regions (3.10) and (3.11) is nonempty for each sufficiently large $\beta$. A simple example is $L_{0}(\beta)=L_{1}(\beta)=\beta^{k}$, which is moreover the choice with about the minimal growth that allows one to draw conclusions about the first $k$ coefficients of the expansion. Of course any choice for $L_{0}(\beta)$ and $L_{1}(\beta)$ growing faster than $\beta^{k}$ (and such that the intersection of the regions (3.10) and (3.11) is nonempty) is also sufficient. For example a region containing the path

$$
L(\beta)=\exp \left(\ln ^{2} \beta\right)
$$


would be convenient, because it allows one to cover all $k$ simultaneously in the above argument. However one should keep in mind that the faster the upper boundary $L_{1}(\beta)$ grows, the more difficult it may be to establish the bound (3.9) within the region (3.10).

A second comment concerns the relation to David's theorem. According to (the lattice counterpart) of this result one expects that the infrared divergent terms cancel in the perturbative coefficients. However, to directly prove such cancellations in the remainder $R_{k}^{\alpha}(\beta, L)$ (e.g., $p(k)=0$ in eq. (3.9)) is probably a more difficult task. The point of using the correlation inequalities is twofold: First, the claim (i) can be established without assuming the IR finiteness of the remainders for fixed $\beta$, as described before. And second, once claim (i) is established, the IR finiteness of the remainders actually follows from the correlation inequality (3.2). To see the latter let $L(\beta)$ be an unbounded path in the intersection of the regions $(3.10),(3.11)$ and let $\left(\beta, L^{\prime}\right)$ be any point in the region $\left\{\left(\beta, L^{\prime \prime}\right) \mid \beta>\beta_{0}, L^{\prime \prime}>L(\beta)\right\}$. Then by (3.2)

$$
\begin{gathered}
A_{k}^{\text {free }}(\beta, L(\beta))+R_{k}^{\text {free }}(\beta, L(\beta)) \leq A_{k}^{\text {free }}\left(\beta, L^{\prime}\right)+R_{k}^{\text {free }}\left(\beta, L^{\prime}\right) \leq R_{k}^{\infty}(\beta) \\
\leq A_{k}^{\operatorname{Dir}}\left(\beta, L^{\prime}\right)+R_{k}^{\operatorname{Dir}}\left(\beta, L^{\prime}\right) \leq A_{k}^{\operatorname{Dir}}(\beta, L(\beta))+R_{k}^{\operatorname{Dir}}(\beta, L(\beta)) .
\end{gathered}
$$

Since, given eq. (3.9), we know the behavior $o\left(\beta^{-k}\right)$ for the terms with $L(\beta)$ and by eq. (3.5) also for $A_{k}^{\alpha}\left(\beta, L^{\prime}\right)$, it follows that $R_{k}^{\alpha}\left(\beta, L^{\prime}\right)=o\left(\beta^{-k}\right)$ is valid "uniformly" in the region $L^{\prime} \geq L(\beta)$. (In other words, the statement is true for arbitrary path $L^{\prime}(\beta)>L(\beta)$ with a bound on $R_{k}^{\alpha}\left(\beta, L^{\prime}(\beta)\right)$ which is independent on how fast $L^{\prime}(\beta)$ grows. Note that this is not uniformity in the usual sense where one would require a region $L^{\prime} \geq L_{0}^{\prime}$ with $L_{0}^{\prime}$ independent of $\beta$.) In a sense this supplements (the expected lattice counterpart of) David's argument on the IR finiteness of the PT coefficients.

It remains to establish eq. (3.9). Since we have not managed to prove this, the proof of (i) and (ii) remains incomplete. However, we find the strategy outlined promising and for the rest of this section we shall give a plausibility argument that eq. (3.9) indeed holds. To explain the argument let us follow the expectation of Patrascioiu and Seiler that PT breaks down for large lattice sizes $L$ since the spins at large relative distances become strongly decorrelated. Consequently, one can question the validity of the bound (3.9) in the whole $L-\beta$ plane, in particular for $\beta$ fixed, $L \rightarrow \infty$. Recall, however, that in the strategy we used to establish claim (i) exclusively lattice sizes of type (3.13) enter which grow with $\beta$ only in lattice units - the 
physical size of these lattices $L(\beta) / \xi(\beta)$ decreases as $\beta \rightarrow \infty$. (Here $\xi(\beta)$ is the correlation length growing exponentially in the standard scenario.) Consequently, in our stratgey it is sufficient to establish the bound (3.9) in the region (3.10) of the $L-\beta$ plane. As it is shown below, for such mildly growing volumes the spins become increasingly ordered with growing $\beta-$ hence the above objection does not apply. Roughly speaking, the argument estimates the probability of having fluctuations $\vec{\pi}_{x}^{2} \geq \delta^{2}$ at least at one of the sites and finds that such fluctuations are exponentially suppressed by a factor $\sim \exp \left(-\pi \delta^{2} \beta / \ln L\right)$.

We begin by considering the effect of constraining the fluctuations in an auxiliary system. Let us calculate the probability that in a massless free theory the fluctuation exceeds some given threshold $\delta$. We shall use again the $\Lambda \cup \partial \Lambda$ square lattice of section 2 and the action

$$
\mathcal{A}_{0}(\phi)=\frac{1}{2} \sum_{x, \mu}\left(\nabla_{\mu} \phi_{x}\right)^{2}
$$

In the case of the Dirichlet BC the field at the boundary is fixed to zero, while for the free $\mathrm{BC}$ we use the remaining global translational symmetry to fix, say $\phi_{0} \equiv \phi(x=0)=0$. (For this BC the site $x=0$ is assumed to be left out in the sums and products appearing in the expressions below.) Consider now the constrained model described by the partition function

$$
Z(\beta, \delta)=\int_{-\delta}^{\delta} \prod_{x \in \Lambda} d \phi_{x} e^{-\beta \mathcal{A}_{0}(\phi)}
$$

Here the field values are restricted to $\left|\phi_{x}\right| \leq \delta$. Define the correction term $\bar{R}(\beta, \delta)$ as

$$
Z(\beta, \delta)=Z(\beta)[1-\bar{R}(\beta, \delta)],
$$

where $Z(\beta)=Z(\beta, \infty)$ is the corresponding partition function for the unrestricted case. The term $\bar{R}(\beta, \delta)$ can be interpreted as the probability to have $\left|\phi_{x}\right|>\delta$, for at least one of the sites $x$. It satisfies the inequality:

$$
\begin{aligned}
& 0 \leq \bar{R}(\beta, \delta) \leq \frac{1}{Z(\beta)} \sum_{x \in \Lambda} \int_{\left|\phi_{x}\right| \geq \delta} d \phi_{x} \int_{-\infty}^{\infty} \prod_{y \neq x} d \phi_{y} e^{-\beta \mathcal{A}_{0}(\phi)} \\
& =\frac{1}{Z(\beta)} \sum_{x \in \Lambda} \frac{1}{2 \pi i} \int_{-\infty}^{\infty} d \alpha\left(\frac{e^{i \alpha \delta}}{\alpha+i 0}-\frac{e^{-i \alpha \delta}}{\alpha-i 0}\right) \int_{-\infty}^{\infty} \prod_{y} d \phi_{y} e^{i \alpha \phi_{x}-\beta \mathcal{A}_{0}(\phi)}
\end{aligned}
$$




$$
\begin{aligned}
& =\sum_{x \in \Lambda} \frac{1}{2 \pi i} \int_{-\infty}^{\infty} d \alpha\left(\frac{e^{i \alpha \delta}}{\alpha+i 0}-\frac{e^{-i \alpha \delta}}{\alpha-i 0}\right) \exp \left(-\frac{D_{x x} \alpha^{2}}{2 \beta}\right) \\
& =\sum_{x \in \Lambda} \exp \left(-\frac{\beta \delta^{2}}{2 D_{x x}}\right) F\left(\delta \sqrt{\frac{\beta}{D_{x x}}}\right) .
\end{aligned}
$$

Here $D_{x x}=\left\langle\phi_{x}^{2}\right\rangle$. The function $F$ is given by

$$
F(\delta)=\frac{1}{\pi} \int_{-\infty}^{\infty} d u e^{-u^{2} / 2} \frac{\delta}{u^{2}+\delta^{2}},
$$

and has the properties $F(0)=1, F(\delta) \leq 1$, and $F(\infty)=0$. Finally, one has

$$
0 \leq \bar{R}(\beta, \delta) \leq|\Lambda| \exp \left(-\frac{\beta \delta^{2}}{2 D_{\max }}\right),
$$

where $D_{\max }=\max _{x} D_{x x}$. In two dimensions $D_{\max } \sim(2 \pi)^{-1} \ln L$ hence

$$
0 \leq \bar{R}(\beta, \delta) \leq L^{2} \exp \left(-\frac{\pi \beta \delta^{2}}{\ln L}\right) .
$$

In fact, $\delta^{2} / 2 D_{x x}$ is the minimal value of the action under the condition that $\phi_{x}=\delta$. To show this consider the configuration

$$
\phi_{y}=\frac{D_{x y}}{D_{x x}} \delta \quad \text { for any } y .
$$

Obviously, $\phi_{x}=\delta$ and it satisfies the lattice equations of motion, $\Delta \phi_{y}=0$ for any $y \neq x$. The corresponding action value is given by

$$
-\frac{1}{2} \sum_{y} \phi_{y}(\Delta \phi)_{y}=\frac{\delta^{2}}{2 D_{x x}}
$$

as stated above. It is also easy to show that the $n$-point functions in the constrained model will differ from those in the unconstrained one also by exponentially small terms of the form (3.19).

We now return to the $\mathrm{O}(n) \sigma$ model. Again, for the case of the free BC's we use the global $\mathrm{O}(n)$ symmetry to fix $\mathbf{S}_{0}=(\overrightarrow{0}, 1)$. Using the parametrization $\mathbf{S}_{x}=\left(\vec{\pi}_{x}, \pm \sqrt{1-\vec{\pi}_{x}^{2}}\right)$ it is easy to verify the following inequality:

$$
1-\mathbf{S}_{x} \cdot \mathbf{S}_{y} \geq \frac{1}{2}\left(\vec{\pi}_{x}-\vec{\pi}_{y}\right)^{2}
$$

\footnotetext{
${ }^{4}$ Note that for $S^{n}>0$ the parametrization $\mathbf{S}=(\vec{\phi}, 1) / \sqrt{1+\vec{\phi}^{2}}$ gives an upper bound, $1-\mathbf{S}_{x} \cdot \mathbf{S}_{y} \leq \frac{1}{2}\left(\vec{\phi}_{x}-\vec{\phi}_{y}\right)^{2}$, which also might be useful.
} 
As a consequence of eq. (3.22), the standard action of the $\sigma$ model is bounded from below by the corresponding free field action, $\mathcal{A}(\mathbf{S}) \geq \mathcal{A}_{0}(\vec{\pi})$ and

$$
\mathcal{A}(\mathbf{S}) \geq \frac{\delta^{2}}{2 D_{x x}} \quad \text { for } \quad\left|\vec{\pi}_{x}\right| \geq \delta .
$$

From here it follows that the probability to have $\left|\vec{\pi}_{x}\right|>\delta$ at least at one site $x$ is suppressed by the exponential factor given in eqs. (3.18) and (3.19). Once again, the constraint influences the correlation functions only by an exponentially small amount as $\beta \rightarrow \infty$. As anticipated, increasing the lattice size not too fast, for example, as in eq. (3.13), the correlation functions of the constrained and unconstrained systems will differ by correction terms which decrease faster than any inverse power of $\beta$. Further the bound (3.19) allows one to choose a constraint $\delta$ which decreases with increasing $\beta$ not too fast, say as $\delta(\beta)^{2} \sim \ln ^{3} \beta \ln L / \beta$. In this case the bound still vanishes faster than any inverse power of $\beta$, while the quantity $\beta \vec{\pi}^{4}$, the leading perturbative term in $\beta \mathcal{A}(\mathbf{S})$, goes to zero as $\beta \rightarrow \infty$.

By comparing the constrained and unconstrained systems one concludes that the fluctuations in the unconstrained system are essentially bounded as $\vec{\pi}_{x}^{2} \leq$ const $\ln L / \beta$. In other words a system of mildly growing size $L(\beta)$ (e.g., as in eq. (3.13)) becomes increasingly ordered as $\beta \rightarrow \infty$. As shown before, within our strategy it is sufficient to prove the existence of the bound (3.9) for such mildly growing $L(\beta)$. By the above reasoning this should be a simpler task, but still remains to be done.

\section{Discussion}

Let us first discuss the physical picture behind the arguments of the previous section in more detail. In [1] the so-called superinstanton configurations are introduced. In analogy with the free field case one considers the configuration with minimal action under the condition that the spin at the middle (at $\left.x=z_{0}\right)$ is rotated by an arbitrary angle relative to the spins at the boundary (fixed by the Dirichlet $\mathrm{BC}$ ). The action value of these configurations is $\mathrm{O}(1 / \ln L)$ in two dimensions, so that they can be viewed as saturating the bound $(3.23)$. They play a crucial role in disordering the system in the infinite volume. As the authors note, the fact that the energy of superinstantons goes to zero as $L \rightarrow \infty$ implies that, in an infinite volume, 
they are present at arbitrarily large $\beta$ and disorder the spins forbidding a spontaneous magnetization in two dimensions. With the assumptions made we have shown, however, that for establishing the correctness of the perturbative expansion it is enough to consider a mildly growing size with $L(\beta)$ given, e.g., by eq. (3.13). Under such circumstances the superinstantons with $\left|\vec{\pi}_{x}\right|>\delta$ are exponentially suppressed and the error made by restricting the integration region to $\left|\vec{\pi}_{x}\right| \leq \delta$ is exponentially small. (After the standard PT steps - rescaling $\vec{\pi}_{x}$ and expanding the Boltzmann factor with only the quadratic part kept in the exponent - the dependence on $\delta$ is again exponentially suppressed, as expected.) For the correlation function $\left\langle S_{x} \cdot S_{y}\right\rangle$ only superinstantons with size smaller than $|x-y|$ contribute significantly, larger ones will rotate the two spins simultaneously. This contribution is responsible for the leading order bare PT result

$$
\left\langle\left(\mathbf{S}_{x}-\mathbf{S}_{0}\right)^{2}\right\rangle \sim \frac{1}{\beta} \frac{N}{2 \pi} \ln |x| .
$$

This also explains why the $\mathrm{O}(\mathrm{n})$ invariant quantities are infrared finite in $\mathrm{PT}$, as opposed to the noninvariant ones which diverge as some power of $\ln L$. However the form of the finite size corrections is incorrectly given by PT, eq. (3.5). Indeed, due to the nonperturbatively generated mass the corrections should be exponentially small for $L \gg \xi(\beta)$.

In $d=1$ dimensions the status of $\mathrm{PT}$ is quite different. In one dimension $D_{\max } \propto L$ and the correction term is of the form $\exp \left(-c \beta \delta^{2} / L\right)$. Consequently, the $\mathrm{O}(1 / L)$ corrections in eq. (3.5) are not negligible compared to higher order terms in $1 / \beta$ even for such large sizes when the perturbative expansion breaks down, i.e., $L(\beta) \propto \beta$. This is in accordance with the observation by Hasenfratz [10] that the limits $\beta \rightarrow \infty$ and $L \rightarrow \infty$ in $d=1$ are not interchangeable. As pointed out in [11], starting from order $\beta^{-3}$ the coefficients are infrared divergent.

The case of the SI BC in $d=2$ is very similar to the $d=1$ case with the usual BC. Indeed, already the tree level result has a finite size correction $\mathrm{O}(1 / \ln L)$ which is comparable to higher order terms even for exponentially large $L(\beta)$ where PT breaks down. The correlation inequalities for this case read

$$
C^{\infty}(x, y ; \beta) \leq C^{\mathrm{Dir}}(x, y ; \beta, L) \leq C^{\mathrm{SI}}(x, y ; \beta, L) .
$$

The first few terms of the perturbative expansion for the nearest neighbors 
are given by 沺

$$
\begin{aligned}
C^{\mathrm{SI}}\left(z_{0}, z_{1} ; \beta, L\right)=1 & -\frac{N}{\beta}\left[\frac{1}{4}-\frac{\pi}{16 \ln L}+\mathrm{O}\left(\frac{1}{\ln ^{2} L}\right)\right] \\
& -\frac{N}{\beta^{2}}\left[\frac{N}{48}+\frac{1}{96}+\mathrm{O}\left(\frac{1}{\ln L}\right)\right]+\ldots
\end{aligned}
$$

while the corresponding piece of $C^{\infty}$ is

$$
C^{\infty}=1-\frac{N}{4 \beta}-\frac{N}{32 \beta^{2}}+\ldots
$$

Clearly, eq. (4.2) is inconsistent with the correlation inequality (4.1) for $N>1$ (i.e., $n=N+1>2$ ) when the formal $L \rightarrow \infty$ limit is taken. In the regime $\ln L(\beta) \leq \ln \xi(\beta) \propto \beta$, however, the $1 / \ln L$ correction term in the tree level contribution restores the inequality.

What is the origin of the anomalously large finite size correction for the SI BC? Consider a general SI BC where the spin $\mathbf{S}_{z_{0}}$ is fixed to a direction which is not necessarily parallel to the spins on the boundary $\partial \Lambda$. Denoting the corresponding expectation value by $\langle\mathcal{O}\rangle_{\mathbf{S}_{z_{0}}}$, the following relation holds

$$
\langle\mathcal{O}\rangle_{\text {Dir }}=\frac{1}{Z(\beta)} \int d \mathbf{S}_{z_{0}}\langle\mathcal{O}\rangle_{\mathbf{S}_{z_{0}}} e^{-\beta F\left(\mathbf{S}_{z_{0}}\right)},
$$

where $F\left(\mathbf{S}_{z_{0}}\right)$ is the free energy of a superinstanton and $Z(\beta)$ is the partition function for Dirichlet BC In the lowest order in $1 / \beta$ and for $\left|\vec{\pi}_{z_{0}}\right| \ll 1$ the free energy is up to a (for our purposes irrelevant) additive constant given by (cf. eq. (3.21))

$$
F\left(\mathbf{S}_{z_{0}}\right)=\frac{1}{2 D\left(z_{0}, z_{0}\right)} \vec{\pi}_{z_{0}}^{2} .
$$

The SI solution gives (cf. eq. (3.20))

$$
\vec{\pi}_{z_{0}}-\vec{\pi}_{z_{1}} \approx \frac{1}{4 D\left(z_{0}, z_{0}\right)} \vec{\pi}_{z_{0}}
$$

Then the integration over the SI solutions (still without the contribution from the fluctuations) introduces a nontrivial expectation value for $\mathbf{S}_{z_{0}} \cdot \mathbf{S}_{z_{1}}$ given by

$$
1-\frac{N \pi}{16 \beta \ln L}+\mathrm{O}\left(\frac{1}{\beta \ln ^{2} L}\right) .
$$

\footnotetext{
${ }^{5} \mathrm{~A}$ curious point in the terminology is that the SI BC with $\mathbf{S}_{z_{0}}=(\overrightarrow{0}, 1)$ is the one which excludes superinstantons centered at $z_{0}$. Rather the ordinary BC takes care of all superinstantons.
} 
The analogous correction term in eq. (4.2) — which is a result of the fluctuations for the case when $\mathbf{S}_{z_{0}}$ is fixed - compensates the large finite size correction in eq. (4.7), as it should, since no such term appears for the Dirichlet BC, eq. (2.6). As eq. (4.5) shows, the natural expansion parameter by integrating over the SI directions is $\ln L / \beta$, and it is easy to see that in generic $\mathrm{O}(N+1)$ models the higher order contributions in eq. 4.7) will be of the form $1 / \ln ^{2} L \cdot(\ln L / \beta)^{k}$. As a consequence, a logarithmic divergence in order $1 / \beta^{3}$ is expected also in the perturbative result with the SI BC, in agreement with the explicit computation of section 2 . Since only the coefficient of the leading power in $N$ has been computed one cannot exclude that for a specific $N$ the divergence is cancelled against one from a subleading power in $N$. In particular it would be interesting to see what happens in the $\mathrm{O}(2)$ model.

So far we mainly considered bare lattice PT in volumes whose physical size goes to zero in the continuum limit, $L(\beta) / \xi(\beta) \rightarrow 0$ as $\beta \rightarrow \infty$. However, this was sufficient, together with the correlation inequalities, to argue that the standard bare PT provides the correct asymptotic expansion for the system in an infinite lattice. The physically more relevant question is whether the renormalized PT provides an asymptotic expansion for the correlation function at short physical distances, $x_{\text {phys }}=\epsilon a \xi, 0<\epsilon \ll 1$. A positive answer to this question is not an automatic consequence of the proposed validity of the bare lattice PT.

Our considerations did not make use of the "integrability" of the $\mathrm{O}(n)$ models. Assuming asymptotic freedom one can establish the existence of a Yangian algebra of non-local conserved charges and the absence of particle production [12, 13]. The $\mathrm{O}(n)$ symmetry then basically determines the $S$ matrix amplitudes 14. This bootstrap $S$-matrix has been tested (at low energies) in lattice studies [15] and used as an input for the thermodynamic Bethe ansatz to compute the exact $m / \Lambda$ ratio [16]. The results are also consistent with the $1 / n$ expansions [17]. Finally one can use the bootstrap $S$-matrix as an input for the form factor approach [18, 19] which provides an alternative nonperturbative definition of the theory. For the $\mathrm{O}(3)$ model the results in [20] strongly indicate that the model thus constructed coincides with the continuum limit of the lattice theory at least at low energies. At intermediate energies the results coincide with renormalized PT and at high energies they are consistent with asymptotic freedom. Two non-perturbative constants determined exactly in this approach [21] are again consistent with 
Monte Carlo data 22, 23, 21]. One cannot help feeling that the most natural way to reconcile these facts is the conventional wisdom. That is the continuum limit of the lattice theory coincides with the model described by the bootstrap approach, which is in turn correctly described by an asymptotically free PT at high energies.

Acknowledgements Firstly we would like to express our particular thanks to Alan Sokal for numerous discussions and helpful comments, and for sending us his proof of a related theorem (see below). We would also like to thank Peter Hasenfratz, Adrian Patrascioiu and Erhard Seiler for useful discussions. M.N. acknowledges support by the Reimar Lüst fellowship of the Max-Planck-Society. A first version of this paper stimulated the response hep-lat/9702008 by A. Patrascioiu and E. Seiler, which in turn motivated us to improve on our exposition.

Note added A concise alternative to our first exposition is also contained in a recent note by Sokal [\$]. In particular he observes that the assumption (3.5) on the finite size dependence of the coefficients can be relaxed as follows: Assume that there exist coefficients $\left\{\bar{c}_{r}\right\}_{r=1}^{k}$ and powers $\epsilon(r)>0$ such that

$$
\left|c_{r}^{\alpha}(L)-\bar{c}_{r}\right| \leq O\left(L^{-\epsilon(r)}\right), \quad \alpha=\text { free, Dir }
$$

for $r=1, \ldots, k$. Further replace the condition on $L_{0}(\beta)$ in eq. (3.10) by $\beta^{k-r} L_{0}(\beta)^{-\epsilon(r)} \rightarrow 0$ for $r=1, \ldots, k$. He assumes that the bound (3.9) has been established in the whole $L-\beta$ plane, but this could also be weakened along the lines described in section 3. Thus assuming that the bound (3.9) has been established for at least one unbounded path contained in the new region (3.10) he shows that $C^{\infty}(\beta)$ has an asymptotic expansion with the coefficients $\bar{c}_{r}$. The proof remains essentially the same: One takes an $L(\beta)$ for which both $A_{k}^{\alpha}$ and $R_{k}^{\alpha}$ are $o\left(\beta^{-k}\right)$ under the assumptions stated and concludes $R_{k}^{\infty}(\beta)=o\left(\beta^{-k}\right)$ from the correlation inequality (3.1).

\section{References}

[1] A. Patrascioiu and E. Seiler, in Fields and Particles, Schladming, 1990, edited by H. Mitter and W. Schweiger (Springer, Berlin, 1990) 
"The Difference between the Abelian and Non-Abelian models: Facts and Fancy", MPI-Ph 91-88 (unpublished).

[2] A. Patrascioiu and E. Seiler, Nucl. Phys. B (Proc. Suppl.) 30 (1993) 184.

[3] J. Fröhlich and T. Spencer, Commun. Math. Phys. 81 (1981) 527.

[4] A. Patrascioiu and E. Seiler, Phys. Rev. Lett. 74 (1995) 1920.

[5] N.D. Mermin and H. Wagner, Phys. Rev. Lett. 17 (1966) 1133.

N.D. Mermin, J. Math. Phys. 8 (1967) 1061.

[6] F. David, Phys. Rev. Lett. 75 (1995) 2626.

[7] A. Patrascioiu and E. Seiler, Phys. Rev. Lett. 75 (1995) 2627.

[8] A. D. Sokal, "Some unquestionable arguments on PT", unpublished note, March '97.

[9] F. David, Commun. Math. Phys. 81 (1981) 149.

[10] P. Hasenfratz, Phys. Lett. B141 (1984) 385.

[11] E. Brezin, F. David and J. Zinn-Justin, unpublished letter, 1985

[12] M. Lüscher, Nucl. Phys. B135 (1978) 1.

[13] D. Buchholz and J.T. Lopuszanski, Lett. Math. Phys. 3 (1979) 175.

D. Bernard, Commun. Math. Phys. 137 (1991) 191.

[14] A.B. and Al.B. Zamolodchikov, Nucl. Phys. B133 (1978) 525.

[15] M. Lüscher and U. Wolff, Nucl. Phys. B339 (1990) 222.

[16] P. Hasenfratz and F. Niedermayer, Nucl. Phys. B414 (1994) 785.

[17] M. Campostrini and P. Rossi, Riv. Nuovo Cimento 16 (1993) 1. M. Campostrini, A. Pelissetto, P. Rossi and E. Vicari, Phys. Rev. B54 (1996) 7301

[18] M. Karowski and P. Weisz, Nucl. Phys. B139 (1978) 455.

[19] F.A. Smirnov, Form Factors in Completely Integrable Models of Quantum Field Theory (World Scientific, Singapore, 1992). 
[20] J. Balog and M. Niedermaier, Nucl. Phys. B500 (1997) 422.

J. Balog, T. Hauer and M. Niedermaier, Phys. Lett. B386 (1996) 224.

[21] J. Balog and M. Niedermaier, Phys. Rev. Lett. 78 (1997) 4151.

[22] B. Alles, A. Buonanno and G. Cella, Nucl. Phys. B500 (1997) 513.

[23] M. Campostrini, A. Pelissetto, P. Rossi and E. Vicari, Phys. Lett. 402 (1997) 141. 